\title{
VARIABILIDAD DE COSTOS DE ANTIPSICÓTICOS SEGÚN ESTABLECIMIENTOS FARMACÉUTICOS EN LIMA, PERÚ
}

\author{
Rubén Valle (101,2,a \\ 1 Centro de Investigación en Epidemiología Clínica y Medicina Basada en Evidencias, Facultad de Medicina Humana, \\ Universidad de San Martín de Porres, Lima, Perú. \\ 2 DEIDAE de Adultos y Adultos Mayores, Instituto Nacional de Salud Mental «Honorio Delgado - Hideyo Noguchi», \\ Lima, Perú. \\ a Médico especialista en psiquiatría, maestro en Ciencias en Investigación Epidemiológica.
}

\section{RESUMEN}

Los objetivos del estudio fueron determinar la variabilidad de costos de antipsicóticos en establecimientos farmacéuticos públicos (hospitales) y privados (farmacias y clínicas), calcular la variabilidad de costos de antipsicóticos entre establecimientos y estimar el costo de tratamiento mensual de mantenimiento con antipsicóticos. Se realizó un estudio de análisis de costos, los costos unitarios de los antipsicóticos se obtuvieron del Observatorio Peruano de Productos Farmacéuticos. Los resultados muestran que la variabilidad de costos de los antipsicóticos fue mayor en farmacias y clínicas que en hospitales, y el análisis de variabilidad de costos entre establecimientos farmacéuticos mostró que el costo de un antipsicótico en una farmacia y clínica fue 1,3 a 140 veces y de 2,8 a 124 veces, respectivamente, el costo que tuvo el fármaco en un hospital. El costo de tratamiento mensual de mantenimiento varió de $\mathrm{S} / 3$ a S/ 2130 según el fármaco y establecimiento farmacéutico.

Palabras clave: Costos y Análisis de costo; Antipsicóticos; Esquizofrenia; Trastornos Mentales; Perú (fuente: DeCS BIREME).

\section{COST VARIABILITY OF ANTIPSYCHOTICS ACCORDING TO PHARMACEUTICAL ESTABLISHMENTS IN LIMA, PERU}

\section{ABSTRACT}

The objectives of the study were to determine the cost variability of antipsychotics in public (hospitals) and private pharmaceutical establishments (pharmacies and clinics), calculate the cost variability of antipsychotics between establishments and estimate the cost of monthly maintenance treatment with antipsychotics. A cost analysis study was performed, unit costs of antipsychotics were obtained from the Peruvian Pharmaceutical Products Observatory. The results show that the cost variability of antipsychotics was greater in pharmacies and clinics than in hospitals, and the analysis of cost variability between pharmaceutical establishments showed that the cost of an antipsychotic in a pharmacy and clinic was 1.3 to 140 times and 2.8 to 124 times, respectively, the cost of the drug in a hospital. The cost of monthly maintenance treatment varied from S/ 3 to S/2130 according to the drug and pharmaceutical establishment.

Citar como: Valle R. Variabilidad de costos de antipsicóticos según establecimientos farmacéuticos en Lima, Perú. 2020;37(1):67-73. Doi: https://doi.org/10.17843/ rpmesp.2020.371.4899

Correspondencia: Rubén Valle; Av. Alameda del Corregidor 1531, La Molina, Lima 15024, Perú; ruben vr12@hotmail.com; (511) 365-2300

Recibido: $20 / 10 / 2019$ Aprobado: $12 / 02 / 2020$

En línea: 23/03/2020
Keywords: Costs and Cost analysis; Antipsychotic Agents; Schizophrenia; Mental Disorders; Peru (source: MeSH NLM).

\section{INTRODUCCIÓN}

Los antipsicóticos son un grupo de fármacos usados en el tratamiento de distintos trastornos mentales. Estas medicinas son la piedra angular del tratamiento de los trastornos psicóticos ${ }^{(1)}$, y algunos de ellos se han aprobado para el tratamiento del trastorno bipolar ${ }^{(2)}$, o se usan de manera no aprobada en el trastorno obsesivo compulsivo y trastorno de personalidad ${ }^{(3,4)}$. Los antipsicóticos son igualmente eficaces en reducir los síntomas de psicosis y se diferencian en el perfil de efectos secundarios que producen ${ }^{(5)}$. Estos fármacos deben ser tomados diariamente (orales) o ser aplicados cada cierto tiempo (depósito) para evitar recaídas y promover la recuperación ${ }^{(6,7)}$. Por ello es importante que los sistemas de salud puedan asegurar el acceso a estas medicinas. 
Los usuarios de antipsicóticos, afiliados al Seguro Integral de Salud (SIS), reciben las medicinas gratuitamente en los establecimientos del Ministerio de Salud (MINSA). Sin embargo, el acceso a estos fármacos en instituciones del MINSA es limitado ya que solo el $78 \%$ de institutos, $64 \%$ de hospitales, $8 \%$ de centros y $1 \%$ de puestos de salud cuentan con antipsicóticos ${ }^{(8)}$. Esta situación dirige a que los pacientes compren sus medicinas en farmacias privadas a un precio mayor, con el consecuente aumento del gasto de bolsillo ${ }^{(8)}$, que recaigan, producto de reducir o dejar de tomar la medicina al no poder comprarlas. Las recaídas clínicas, a su vez, conllevan a mayores gastos para el sistema de salud por el uso de servicios más costosos como de emergencia u hospitalización ${ }^{(9)}$.

Los costos de los antipsicóticos varían ampliamente en el mercado. La variabilidad del costo (relación entre el costo mínimo y máximo) de la risperidona en la India es de 1 a 16 y de la olanzapina de 1 a 12; la variabilidad de estos fármacos en Brasil es de 1 a 35000 y de 1 a 79 , respectivamente ${ }^{(10,11)}$. El alto costo que pueden alcanzar los antipsicóticos genera un menor acceso a estas medicinas y la posibilidad de que los pacientes no puedan continuar con el tratamiento. En Perú, un reporte mostró amplias variaciones en los costos de las medicinas, pudiendo ser el costo de una medicina diez veces más en una farmacia privada que en una pública ${ }^{(12)}$. Sin embargo, la variabilidad de los costos de los antipsicóticos no ha sido estudiada específicamente.

Los objetivos del estudio son determinar la variabilidad de costos de antipsicóticos en establecimientos farmacéuticos públicos (hospitales) y privados (farmacias y clínicas), calcular la variabilidad de costos de antipsicóticos entre establecimientos farmacéuticos y estimar el costo de tratamiento mensual de mantenimiento con antipsicóticos en monoterapia.

\section{EL ESTUDIO}

Se realizó un estudio económico parcial de tipo análisis de costos según la perspectiva del usuario. Los costos unitarios de los antipsicóticos se obtuvieron del Observatorio Peruano de Productos Farmacéuticos (OPPF) de la Dirección General de Medicamentos, Insumos y Drogas (DIGEMID), donde los establecimientos farmacéuticos colocan información sobre los costos de las medicinas ${ }^{(13)}$. El nombre genérico de los antipsicóticos del Petitorio Nacional Único de Medicamentos Esenciales de Perú y de otros antipsicóticos usados en la atención psiquiátrica se introdujo en el buscador ${ }^{(14)}$. La búsqueda brindó información sobre el establecimiento farmacéutico, fecha de actualización de los datos, nombre del producto, laboratorio, costo unitario e información técnica del producto.

Los establecimientos farmacéuticos públicos de las farmacias de hospitales del MINSA y de la DIGEMID se categorizaron en «hospitales»; los establecimientos farmacéuticos de boticas y farmacias privadas en «farmacias»; y los estable-

\section{MENSAJES CLAVE}

Motivación para realizar el estudio: Aunque los antipsicóticos sirven para el manejo de varios trastornos mentales y se deben tomar por largo tiempo, su costo en el mercado puede ser alto y dificultar el acceso a estas medicinas.

Principales hallazgos: El costo unitario y tratamiento mensual con antipsicóticos es mayor en farmacias y clínicas que en hospitales. El costo de tratamiento mensual varía de S/ 3 a S/ 2130 según el fármaco y establecimiento farmacéutico.

Implicancias: Se debe asegurar el abastecimiento con antipsicóticos en los hospitales públicos para que los usuarios de antipsicóticos no tengan problemas en el acceso a estas medicinas.

cimientos farmacéuticos de clínicas privadas en «clínicas». Además, los antipsicóticos cuyo nombre correspondía a la denominación común internacional fueron categorizados como "genéricos»; aquellos cuyo nombre correspondía al fármaco estudiado en investigaciones farmacológicas y que obtuvo la patente como «innovadores»; y los antipsicóticos que figuraban con un nombre comercial pero diferente al del innovador como "genéricos de marca» ${ }^{(15)}$.

El análisis descriptivo consistió en el cálculo de la mediana y el valor mínimo y máximo del costo del antipsicótico según el tipo de fármaco y establecimiento farmacéutico. El análisis de variabilidad de costo por antipsicótico comparó el costo mínimo y máximo de un antipsicótico de la misma formulación dispensado por el mismo tipo de establecimiento farmacéutico. El análisis de variabilidad de costos entre establecimientos farmacéuticos comparó la mediana del costo de un antipsicótico en un hospital con aquella de una farmacia y una clínica. La estimación del costo de tratamiento mensual de mantenimiento con antipsicóticos en monoterapia se realizó basado en el límite superior del rango de la dosis de mantenimiento de cada antipsicótico según el estudio de consenso internacional sobre dosificación ${ }^{(16)}$. El análisis se realizó con el programa estadístico Stata v12 (StataCorp LP, College Station, TX, EUA).

\section{HALLAZGOS}

Los datos de 11 antipsicóticos estuvieron disponibles en el OPPF al 5 de julio de 2019: seis de primera generación y cinco de segunda. Los datos habían sido ingresados al OPPF entre el 5 de mayo al 4 de julio de 2019. Solo tres antipsicóticos estuvieron disponibles como innovadores: decanoato de haloperidol (Haldol decanoas), quetiapina (Seroquel) y olanzapina (Zyprexa). Los datos fueron reportados mayor- 
mente por farmacias $(77,4 \%-99,9 \%)$, seguido de hospitales (0,1\%-100\%) y clínicas $(0,1 \%-6,5 \%)$ (Tabla 1$)$.

\section{Costos de antipsicóticos}

La mediana de los costos unitarios de los antipsicóticos orales fue menor en hospitales que en farmacias y clínicas. La mediana de los costos de los antipsicóticos en hospitales varió de $\mathrm{S} /$ 0,1 (haloperidol) a S/ 1,3 (aripiprazole), en farmacias de S/ 0,68 (clorpromazina) a S/ 24,0 (olanzapina) y en clínicas de S/ 0,5 (haloperidol) a S/ 38,9 (olanzapina). La mediana de los costos de los antipsicóticos genéricos fue menor que los genéricos de marca (excepto para la risperidona vendida en hospitales), y los costos de estos, que los de los innovadores. Esta tendencia también se observó en el caso del decanoato de haloperidol. El decanoato de flufenazina solo estuvo disponible en hospitales a un costo (mediana) de S/ 9,5 (Tabla 2).

\section{Variabilidad de costos por antipsicótico y entre estableci-} mientos farmacéuticos

La mayor variabilidad de costos en hospitales llegó a ser de 1 a 6,5 en genéricos (clorpromazina) y de 1 a 6 (olanzapina) en los genéricos de marca. En farmacias, la mayor variabilidad en genéricos fue de 1 a 24,5 (risperidona), en genéricos de marca fue de 1 a 59 (olanzapina) y en innovadores de 1 a 1,6 (decanoato de haloperidol). En clínicas, la mayor variabilidad en genéricos fue de 1 a 30 (risperidona), en genéricos de marca fue de 1 a 11 (olanzapina) y en innovadores fue de 1 a 5,1 (olanzapina).
La mayor variabilidad de costos entre hospitales y farmacias en genéricos se registró para el haloperidol (1 a 9) y en genéricos de marca para la risperidona (1 a 140). La variabilidad de costos más alta entre hospitales y clínicas en genéricos fue para la trifluoperazina ( 1 a 20,3) y en genéricos de marca para la risperidona (1 a 124) (Tabla 3 ).

\section{Costo del tratamiento mensual}

El costo del tratamiento mensual de mantenimiento con antipsicóticos fue menor en hospitales que en farmacias y clínicas. El costo de tratamiento mensual varió de S/3 (haloperidol/genérico) a S/144 (levomepromazina/genérico de marca) en hospitales, de S/27 (haloperidol/genérico) a S/ 1560 (olanzapina/ innovador) en farmacias y de $\mathrm{S} / 15$ (haloperidol/genérico) a S/2130 (olanzapina/innovador) en clínicas. El tratamiento en farmacias y clínicas con risperidona, quetiapina y levomepromazina (solo farmacias) en genérico fue más alto que con su genéricos de marca en hospitales. El costo de tratamiento mensual en hospitales con decanoato de haloperidol $(\mathrm{S} / 70,5)$ fue casi el doble que con decanoato de flufenazina (S/ 38) (Tabla 4).

\section{DISCUSIÓN}

El estudio ofrece una perspectiva económica del costo de los antipsicóticos y del costo de tratamiento con estos fármacos. Los resultados muestran que la variación de costos es mayor en establecimientos farmacéuticos privados (farmacias y clí-

Tabla 1. Disponibilidad de antipsicóticos por establecimiento farmacéutico y tipo de fármaco en Lima

\begin{tabular}{|c|c|c|c|c|c|c|c|c|c|c|c|c|c|}
\hline \multirow{3}{*}{ Antipsicóticos } & \multirow{3}{*}{ Total $^{\mathbf{a}}$} & \multicolumn{6}{|c|}{ Establecimiento farmacéutico } & \multicolumn{6}{|c|}{ Tipo de fármaco } \\
\hline & & \multicolumn{2}{|c|}{ Hospital } & \multicolumn{2}{|c|}{ Farmacia } & \multicolumn{2}{|c|}{ Clínica } & \multicolumn{2}{|c|}{ Genérico } & \multicolumn{2}{|c|}{$\begin{array}{c}\text { Genérico de } \\
\text { marca }\end{array}$} & \multicolumn{2}{|c|}{ Innovador } \\
\hline & & $\mathbf{n}$ & $\%$ & $\mathbf{n}$ & $\%$ & $\mathbf{n}$ & $\%$ & $\mathbf{n}$ & $\%$ & $\mathbf{n}$ & $\%$ & $\mathbf{n}$ & $\%$ \\
\hline \multicolumn{14}{|l|}{ Primera generación } \\
\hline Haloperidol & 548 & 12 & 2,2 & 535 & 97,6 & 1 & 0,2 & 548 & 100 & 0 & 0 & 0 & 0 \\
\hline Trifluoperazina & 910 & 9 & 0,9 & 900 & 98,9 & 1 & 0,1 & 910 & 100 & 0 & 0 & 0 & 0 \\
\hline Clorpromozina & 503 & 10 & 1,9 & 485 & 96,4 & 8 & 1,6 & 503 & 100 & 0 & 0 & 0 & 0 \\
\hline Levomepromazina & 62 & 10 & 16,1 & 48 & 77,4 & 4 & 6,5 & 4 & 6,5 & 58 & 93,6 & 0 & 0 \\
\hline Sulpirida & 2007 & 12 & 0,6 & 1985 & 98,9 & 10 & 0,5 & 0 & 0 & 2007 & 100 & 0 & 0 \\
\hline D. de haloperidol & 934 & 10 & 1,1 & 924 & 98,9 & 0 & 0 & 10 & 1,1 & 6 & 0,64 & 918 & 98,9 \\
\hline D. de flufenazina & 3 & 3 & 100 & 0 & 0 & 0 & 0 & 3 & 100 & 0 & 0 & 0 & 0 \\
\hline \multicolumn{14}{|l|}{ Segunda generación } \\
\hline Risperidona & 5069 & 14 & 0,4 & 5034 & 99,3 & 21 & 0,4 & 2714 & 53,5 & 2355 & 46,5 & 0 & 0 \\
\hline Quetiapina & 2883 & 10 & 0,4 & 2869 & 99,9 & 10 & 0,1 & 107 & 3,7 & 2771 & 96,1 & 5 & 0,2 \\
\hline Aripiprazole & 1899 & 1 & 0,1 & 1898 & 99,9 & 0 & 0 & 0 & 0 & 1889 & 100 & 0 & 0 \\
\hline Olanzapina & 2878 & 4 & 0,1 & 2864 & 99,5 & 10 & 0,4 & 0 & 0 & 1971 & 68,5 & 907 & 31,5 \\
\hline Clozapina & 82 & 12 & 14,6 & 68 & 82,9 & 2 & 2,4 & 12 & 14,6 & 70 & 85,4 & 0 & 0 \\
\hline
\end{tabular}

a Establecimientos que dispensan antipsicóticos según el Observatorio Peruano de Productos Farmacéuticos.

D: decanoato. 
Tabla 2. Costo unitario de antipsicóticos según establecimiento farmacéutico y tipo de fármaco en Lima

\begin{tabular}{|c|c|c|c|c|c|c|c|c|c|}
\hline \multirow{3}{*}{ Antipsicóticos } & \multicolumn{9}{|c|}{ Establecimiento farmacéutico $(\mathrm{S} /)^{\mathrm{b}}$} \\
\hline & \multicolumn{3}{|c|}{ Hospital } & \multicolumn{3}{|c|}{ Farmacia } & \multicolumn{3}{|c|}{ Clínica } \\
\hline & Mediana & Mín. & Máx. & Mediana & Mín. & Máx. & Mediana & Mín. & Máx. \\
\hline \multicolumn{10}{|l|}{ Primera generación } \\
\hline \multicolumn{10}{|l|}{ Haloperidol (10 mg) } \\
\hline Genérico & 0,1 & 0,07 & 0,34 & 0,9 & 0,31 & 6,5 & $0,5^{\mathrm{a}}$ & 0,5 & 0,5 \\
\hline \multicolumn{10}{|l|}{ Trifluoperazina (5 mg) } \\
\hline Genérico & 0,36 & 0,31 & 0,39 & 1,3 & 0,15 & 1,6 & $7,3^{\mathrm{a}}$ & 7,3 & 7,3 \\
\hline \multicolumn{10}{|c|}{ Clorpromozina (100 mg) } \\
\hline Genérico & 0,55 & 0,1 & 0,65 & 0,68 & 0,2 & 1,3 & 1,8 & 0,7 & 2,7 \\
\hline \multicolumn{10}{|c|}{ Levomepromazina (100 mg) } \\
\hline Genérico & & & & 2,4 & 2,4 & 2,4 & & & \\
\hline Genérico de marca & 1,2 & 0,55 & 1,2 & 3,1 & 2,7 & 3,8 & 3,3 & 2,9 & 4,4 \\
\hline \multicolumn{10}{|l|}{ Sulpirida (200 mg) } \\
\hline Genérico de marca & 0,15 & 0,1 & 0,2 & 3,9 & 1,9 & 5,7 & 4,4 & 2,1 & 6,7 \\
\hline \multicolumn{10}{|c|}{ D. de haloperidol (50 mg) } \\
\hline Genérico & 23,5 & 23,5 & 26,3 & & & & & & \\
\hline Genérico de marca & - & & - & 49,8 & 45,5 & 54,1 & & & - \\
\hline Innovador & - & & - & 58,4 & 51,9 & 81,9 & & & - \\
\hline \multicolumn{10}{|c|}{ D. de flufenazina (25 mg) } \\
\hline Genérico de marca & 9,5 & 9,5 & 9,6 & & & & & & \\
\hline \multicolumn{10}{|l|}{ Segunda generación } \\
\hline \multicolumn{10}{|l|}{ Risperidona (2 mg) } \\
\hline Genérico & 0,19 & 0,05 & 0,31 & 1,56 & 0,33 & 8,1 & 2,5 & 0,31 & 9,3 \\
\hline Genérico de marca & 0,05 & 0,03 & 0,05 & 7,0 & 1,4 & 12,3 & $6,2^{\mathrm{a}}$ & 6,2 & 6,2 \\
\hline \multicolumn{10}{|l|}{ Quetiapina (200 mg) } \\
\hline Genérico & - & & - & 5,7 & 1,6 & 5,7 & 3,5 & 2,2 & 4,7 \\
\hline Genérico de marca & 0,58 & 0,49 & 2,4 & 9,2 & 1,0 & 19,7 & 7,9 & 1,5 & 10,8 \\
\hline Innovador & - & & - & 13,0 & 12,8 & 13,0 & & & - \\
\hline \multicolumn{10}{|l|}{ Aripiprazole (15 mg) } \\
\hline Genérico de marca & $1,3^{\mathrm{a}}$ & 1,3 & 1,3 & 10,0 & 8,0 & 30,1 & & & \\
\hline \multicolumn{10}{|l|}{ Olanzapina (10 mg) } \\
\hline Genérico de marca & 0,79 & 0,35 & 2,1 & 13,8 & 0,4 & 23,7 & 19,8 & 4,5 & 51,4 \\
\hline Innovador & - & & & 24,0 & 7,0 & 35,5 & 38,9 & 32,1 & 41,6 \\
\hline \multicolumn{10}{|l|}{ Clozapina (100 mg) } \\
\hline Genérico & 0,33 & 0,15 & 0,68 & & & & & & \\
\hline Genérico de marca & - & & - & 6,4 & 5,8 & 7,9 & $7,3^{\mathrm{a}}$ & 7,3 & 7,3 \\
\hline
\end{tabular}

a Solo hubo una observación; ${ }^{\mathrm{b}}$ los costos menores de S/ 1 se representan con dos decimales.

D: decanoato; Mín.: mínimo; Máx.: máximo; guion: no se reportaron datos.

nicas) que en públicos (hospitales). La estrecha variabilidad de costos de antipsicóticos en hospitales se podría deber a la norma que señala que el precio de venta de una medicina en dicho sector no puede exceder el $25 \%$ de su precio de compra ${ }^{(17)}$, mientras que la alta variabilidad del costo de los antipsicóticos en farmacias y clínicas se debería a la no existencia de normas que regulen el costo de fármacos en este sector $^{(18)}$. Así, la distinta regulación entre el sector público y privado explicaría las diferencias en la variabilidad de costos de los antipsicóticos.
Se ha argumentado que el costo mayor en establecimientos privados es porque estos centros venden medicinas «de marca» cuyo costo de compra es mayor que los genéricos ${ }^{(12)}$, y porque el sector público realiza compras corporativas que le permite vender medicinas a bajo costo. Contrariamente, nuestro estudio muestra que algunos antipsicóticos genéricos de marca en hospitales tienen un costo menor que sus genéricos vendidos en farmacias y clínicas. Además, las farmacias privadas en Perú han tenido un proceso de fusión que les permite manejar gran parte del mercado y por lo tanto hacer 
Tabla 3. Variabilidad de costos por antipsicótico y entre establecimientos farmacéuticos en Lima

\begin{tabular}{|c|c|c|c|c|c|}
\hline \multirow[t]{2}{*}{ Antipsicóticos } & \multicolumn{3}{|c|}{$\begin{array}{l}\text { Variabilidad por antipsicótico } \\
\text { (razón entre costo máximo y mínimo) }\end{array}$} & \multicolumn{2}{|c|}{$\begin{array}{c}\text { Variabilidad entre establecimientos } \\
\text { (razón entre medianas) }\end{array}$} \\
\hline & Hospital & Farmacia & Clínica & Farmacia/hospital & Clínica/hospital \\
\hline \multicolumn{6}{|l|}{ Primera generación } \\
\hline \multicolumn{6}{|l|}{ Haloperidol } \\
\hline Genérico & 4,9 & 21,0 & a & 9,0 & 5,0 \\
\hline \multicolumn{6}{|l|}{ Trifluoperazina } \\
\hline Genérico & 1,3 & 10,7 & a & 3,6 & 20,3 \\
\hline \multicolumn{6}{|l|}{ Clorpromozina } \\
\hline Genérico & 6,5 & 6,5 & 3,9 & 1,3 & 3,3 \\
\hline \multicolumn{6}{|l|}{ Levomepromazina } \\
\hline Genérico & & 1,0 & - & & \\
\hline Genérico de marca & 2,2 & 1,4 & 1,5 & 2,6 & 2,8 \\
\hline \multicolumn{6}{|l|}{ Sulpirida } \\
\hline Genérico de marca & 2,0 & 3,0 & 3,2 & 26,0 & 29,3 \\
\hline \multicolumn{6}{|l|}{ D. de haloperidol } \\
\hline Genérico & 1,1 & - & & & - \\
\hline Genérico de marca & & 1,2 & - & & - \\
\hline Innovador & & 1,6 & & - & \\
\hline \multicolumn{6}{|l|}{ D. de flufenazina } \\
\hline Genérico & 1,0 & - & - & & \\
\hline \multicolumn{6}{|l|}{ Segunda generación } \\
\hline \multicolumn{6}{|l|}{ Risperidona } \\
\hline Genérico & 6,2 & 24,5 & 30,0 & 8,2 & 13,2 \\
\hline Genérico de marca & 1,7 & 8,8 & a & 140,0 & 124,0 \\
\hline \multicolumn{6}{|l|}{ Quetiapina } \\
\hline Genérico & - & 3,6 & 2,1 & - & \\
\hline Genérico de marca & 4,9 & 19,7 & 7,2 & 16,0 & 14,0 \\
\hline Innovador & & 1,0 & & & \\
\hline \multicolumn{6}{|l|}{ Aripiprazole } \\
\hline Genérico de marca & a & 3,8 & & 7,7 & \\
\hline \multicolumn{6}{|l|}{ Olanzapina } \\
\hline Genérico de marca & 6,0 & 59,0 & 11,4 & 17,0 & 25,0 \\
\hline Innovador & & 1,3 & 5,1 & - & \\
\hline \multicolumn{6}{|l|}{ Clozapina } \\
\hline Genérico & 5,0 & & & - & \\
\hline Genérico de marca & & 1,4 & 1,0 & - & \\
\hline
\end{tabular}

a Solo hubo una observación.

D: decanoato, Mín.: mínimo, Máx.: máximo; guion: no se reportaron datos.

compras corporativas ${ }^{(18)}$. En ese sentido, el alto costo de los antipsicóticos en farmacias y clínicas parece obedecer exclusivamente a las leyes del mercado y a la búsqueda de mayores márgenes de ganancias.

La estimación del costo de tratamiento mensual de mantenimiento se realizó con base en un esquema de monoterapia cuando la polifarmacia, uso conjunto de más de un psicofármaco, es más común ${ }^{(19)}$. La polifarmacia no está respaldada por la evidencia y agrega mayores costos al tratamiento por la suma de un antipsicótico u otro psicofármaco al esquema terapéutico; sin embargo, es bastante usada en nuestro medio. Por ejemplo, dos estudios en pacientes con esquizofrenia mostraron que el 40,5\% de pacientes de consulta ambulatoria y $57 \%$ de hospitalizados recibía más de un antipsicótico ${ }^{(20,21)}$. Por ello, los costos de tratamiento de nuestro estudio solo aplican para el tratamiento en monoterapia y no para los tratamientos en polifarmacia cuyo costo sería mayor.

El estudio presenta algunas limitaciones. Un reporte de la Defensoría del Pueblo encontró que la coincidencia entre el costo de las medicinas de las farmacias y del OPPF era del $69,3 \%{ }^{(12)}$, por ello es probable que un porcentaje de los costos de los antipsicóticos del OPPF pueda ser diferente a los costos que tienen en las farmacias debido a una falta de 
Tabla 4. Estimado del costo mensual del tratamiento de mantenimiento con antipsicóticos

\begin{tabular}{|c|c|c|c|c|c|c|c|}
\hline \multirow{2}{*}{ Antipsicóticos } & \multirow{2}{*}{$\begin{array}{l}\text { Dosis de mantenimiento } \\
\qquad(\mathrm{mg})^{\mathrm{a}}\end{array}$} & \multicolumn{3}{|c|}{ Costo mensual (S/) } & \multicolumn{3}{|c|}{ Costo mensual (USD) ${ }^{\mathrm{b}}$} \\
\hline & & Hospital & Farmacia & Clínica & Hospital & Farmacia & Clínica \\
\hline \multicolumn{8}{|l|}{ Primera generación } \\
\hline Haloperidol & 10 & & & & & & \\
\hline Genérico & & 3,0 & 27,0 & 15,0 & 0,9 & 8,2 & 4,5 \\
\hline Trifluoperazina & 20 & & & & & & \\
\hline Genérico & & 43,2 & 156,0 & 876,0 & 13,1 & 47,3 & 265,5 \\
\hline Clorpromozina & 600 & & & & & & \\
\hline Genérico & & 99,0 & 122,4 & 324,0 & 30,0 & 37,1 & 98,2 \\
\hline Levomepromazina & 400 & & & & & & \\
\hline Genérico & & & 288,0 & & & 87,3 & \\
\hline Genérico de marca & & 144,0 & 372,0 & 396,0 & 43,6 & 112,7 & 120,0 \\
\hline Sulpirida & 600 & & & & & & \\
\hline Genérico de marca & & 13,5 & 351,0 & 396,0 & 4,1 & 106,4 & 120,0 \\
\hline D. de haloperidol & 150 & & & & & & \\
\hline Genérico & & 70,5 & & & 21,4 & - & - \\
\hline Genérico de marca & & & 149,4 & & & 45,3 & \\
\hline Innovador & & & 155,4 & & & 47,1 & \\
\hline D. de flufenazina & 100 & & & & & & \\
\hline Genérico & & 38,0 & & & 11,5 & & \\
\hline \multicolumn{8}{|l|}{ Segunda generación } \\
\hline Risperidona & 6 & & & & & & \\
\hline Genérico & & 17,1 & 140,4 & 225,0 & 5,2 & 42,5 & 68,2 \\
\hline Genérico de marca & & 4,5 & 360,0 & 558,0 & 1,4 & 190,9 & 169,1 \\
\hline Quetiapina & 800 & & & & & & \\
\hline Genérico & & & 684,0 & 420,0 & & 207,3 & 127,3 \\
\hline Genérico de marca & & 69,6 & 1104,0 & 948,0 & 21,1 & 334,5 & 287,3 \\
\hline Innovador & & & 1560,0 & & & 472,0 & \\
\hline Aripiprazole & 30 & & & & & & \\
\hline Genérico de marca & & 78,0 & 600,0 & & 23,6 & 181,8 & \\
\hline Olanzapina & 20 & & & & & & \\
\hline Genérico de marca & & 47,4 & 828,0 & 1188,0 & 14,4 & 250,9 & 360,0 \\
\hline Innovador & & & 1440,0 & 2130,0 & & 436,4 & 645,5 \\
\hline Clozapina & 500 & & & & & & \\
\hline Genérico & & 49,5 & & & 15,0 & & \\
\hline Genérico de marca & & & 960,0 & 1095,0 & & 290,9 & 331,8 \\
\hline
\end{tabular}

${ }^{a}$ Basado en el estudio de consenso internacional sobre dosificación de antipsicóticos ${ }^{(16)}$. Las dosis no son necesariamente comparables. ${ }^{\mathrm{b}}$ Tipo de cambio: 1 USD= $\mathrm{S} / 3,3$. D: decanoato; guion: no se reportaron datos.

actualización o subreporte por parte de las farmacias. La estimación del costo de tratamiento de mantenimiento mensual fue realizada sobre la base de dosis recomendadas y no a las dosis prescritas, por ello, nuestras estimaciones podrían ser diferentes al costo real del tratamiento. La inferencia de nuestros resultados se podría afectar si es que establecimientos que venden antipsicóticos no las consignen en el OPPF; sin embargo, esta probabilidad es baja ya que solo el 6,2\% de farmacias no están registradas en el OPPF ${ }^{(12)}$. Por otro lado, los datos del OPPF permitieron superar problemas de reticencia por parte de las farmacias de brindar información sobre los costos de las medicinas ${ }^{(22)}$, y permitieron conocer el costo de antipsicóticos de un amplio número de farmacias que de otra manera hubiese tomado altos recursos. 
En conclusión, los resultados muestran que la variabilidad de costos de los antipsicóticos es mayor en farmacias y clínicas que en hospitales, y el análisis de variabilidad de costos entre establecimientos farmacéuticos muestra que el costo de un antipsicótico en una farmacia y clínica puede llegar a ser de 1,3 a 140 veces y de 2,8 a 124 veces el costo que tiene el fármaco en un hospital, respectivamente. El costo de tratamiento mensual con antipsicóticos en monoterapia varía de S/ 3 a S/ 2130 según el tipo de fármaco y establecimiento farmacéutico. La amplia variabilidad de costos de antipsicóticos identificada en nuestro estudio demanda que se tomen algunas medidas. Las autoridades de salud mental deben asegurar el abastecimiento de antipsicóticos en las instituciones del MINSA con fines de que la población usuaria de estos fármacos afiliada al SIS pueda

\section{REFERENCIAS BIBLIOGRÁFICAS}

1. Kreyenbuhl J, Buchanan RW, Dickerson FB, Dixon LB, Schizophrenia Patient Outcomes Research Team (PORT). The Schizophrenia Patient Outcomes Research Team (PORT): updated treatment recommendations 2009. Schizophr Bull. 2010;36(1):94-103. doi: 10.1093/schbul/ sbp130

2. Frye MA. Clinical practice. Bipolar disorder--a focus on depression. $\mathrm{N}$ Engl J Med. 2011;364(1):51-9. doi: 10.1056/NEJMc1101370

3. Zhou D-D, Zhou X-X, Lv Z, Chen X-R, Wang W, Wang G-M, et al. Comparative efficacy and tolerability of antipsychotics as augmentations in adults with treatment-resistant obsessive-compulsive disorder: A network meta-analysis. J Psychiatr Res. 2019;111:51-8. doi: 10.1016/j. jpsychires.2019.01.014

4. Stoffers J, Völlm BA, Rücker G, Timmer A, Huband N, Lieb K. Pharmacological interventions for borderline personality disorder. Cochrane Database Syst Rev. 2010;(6):1-166. doi: 10.1002/14651858. CD005653.pub2

5. Leucht S, Heres S, Kissling W, Davis JM. Evidence-based pharmacotherapy of schizophrenia. Int J Neuropsychopharmacol. 2011;14(2):269-84. doi: 10.1017/S1461145710001380

6. Kishi T, Ikuta T, Matsui Y, Inada K, Matsuda Y, Mishima K, et al. Effect of discontinuation v. maintenance of antipsychotic medication on relapse rates in patients with remitted/stable first-episode psychosis: a meta-analysis. Psychol Med. 2019;49(5):772-9. doi: 10.1017/ S0033291718001393

7. Guo X, Zhang Z, Zhai J, Fang M, Hu M, Wu R, et al. Effects of antipsychotic medications on quality of life and psychosocial functioning in patients with early-stage schizophrenia: 1-year follow-up naturalistic study. Compr Psychiatry. 2012;53(7):1006-12. Doi: 10.1016/j. comppsych.2012.03.003

8. Hodgkin D, Piazza M, Crisante M, Gallo C, Fiestas F. Availability of psychotropic medications in health care facilities of the Ministry of Health of Peru, 2011. Rev Peru Med Exp Salud Publica. 2014;31(4):660-8.

9. Pennington M, McCrone P. The Cost of Relapse in Schizophrenia. PharmacoEconomics. 2017;35(9):921-36. doi: 10.1007/s40273-017-0515-3

10. Shukla AK, Agnihotri A. Cost analysis of antipsychotic drugs available in India. Int J Basic Clin Pharmacol. 2017;6(3):669-74. doi: http://dx.doi. org/10.18203/2319-2003.ijbcp20170834

11. Razzouk D. Cost variation of antipsychotics in the public health system in Brazil. J Bras Econ Saude. 2017;9((Suppl.1)):49-57. doi: 10.21115/ JBES.v9.suppl1.49-57 siempre recibirlas, y que los usuarios no afiliados al SIS puedan comprarlos en estos centros. Además, las autoridades de salud deben valorar la opción de regular el costo de los antipsicóticos en el mercado privado, tal como ocurre en otros países. Finalmente, los médicos que recetan antipsicóticos deberían conocer los costos de estos fármacos, valorar el costo de los antipsicóticos antes de su prescripción y cumplir la norma técnica de recetar medicamentos genéricos.

Contribuciones de autoría: RV concibió y diseño del estudio, recolectó los datos, analizó e interpretó los resultados, redactó el artículo y aprobó la versión final.

Fuentes de financiamiento: Autofinanciado.

Conflictos de interés: El autor declara no tener conflictos de interés.

12. Defensoría del Pueblo. Reporte Derecho a la salud [Internet]. Lima: Defensoría del Pueblo; 2018 [citado el 4 de julio de 2019]. Disponible en: https://www.defensoria.gob.pe/wp-content/uploads/2018/12/Reporte-Derecho-a-la-Salud.pdf

13. Observatorio Peruano de Productos Farmacéuticos [Internet]. Lima: Dirección General de Medicamentos, Insumos y Drogas; 2019 [citado el 5 de julio de 2019]. Disponible en: http://observatorio.digemid.minsa.gob.pe/.

14. Ministerio de Salud. Petitorio Nacional Único de Medicamentos esenciales para el sector salud [Internet]. Lima: Dirección General de Medicamentos, Insumos y Drogas; 2015 [citado el 6 de julio de 2019]. Disponible en: http://www.digemid.minsa.gob.pe/UpLoad/UpLoaded/PDF/Normatividad/2018/RM_1361-2018.pdf

15. Díez MV, Errecalde MF. Aclaraciones al concepto de genérico. Inf Ter Sist Nac Salud. 1998;22(3):68-72.

16. Gardner DM, Murphy AL, O'Donnell H, Centorrino F, Baldessarini RJ. International consensus study of antipsychotic dosing. Am J Psychiatry. 2010;167(6):686-93.

17. Ministerio de Salud. Directiva Administrativa N²49- MINSA/2018/DIGEMID Gestión del sistema integrado de suministro público de productos farmacéuticos, dispositivos médicos y productos sanitarios-SISMED [Internet], 2018 [citado el 7 de julio de 2019]. Disponible en: http://www. digemid.minsa.gob.pe/UpLoad/UpLoaded/PDF/EAccMed/Normatividad/E03_RM_116-2018.pdf

18. Huarag E. ¿Un remedio peor que la enfermedad? sobre concentraciones, monopolios y acceso a los medicamentos. Pluriversidad. 2018;1(1):11126. doi: 10.31381/pluriversidad.vli1.1674

19. Kukreja S, Kalra G, Shah N, Shrivastava A. Polypharmacy in psychiatry: a review. Mens Sana Monogr. 2013;11(1):82-99. doi: 10.4103/0973-1229.104497

20. Stucchi-Portocarrero S, Saavedra JE. Polifarmacia psiquiátrica en personas con esquizofrenia en un establecimiento público de salud mental en Lima. Rev Neuro-Psiquiatr. 2018;81(3):145-53. doi: 10.20453/rnp.v81i3.3382

21. Bojórquez Giraldo E, Arévalo Alván A, Castro Cisneros K, Ludowieg Casinelli L, Orihuela Fernández S. Patrones de prescripción de psicofármacos en pacientes con esquizofrenia y trastornos relacionados internados en el Hospital Víctor Larco Herrera, 2015. An Fac Med. 2017;78(4):386-92. doi: 10.15381/anales.v78i4.14258

22. Miranda J. El mercado de medicamentos en el Perú ¿ilibre o regulado? Lima: Consorcio de Investigación Económica y Social [Internet]. Lima: Ministerio de Economía y Finanzas; 2004 [citado el 10 de julio de 2019]. Disponible en:https://www.mef.gob.pe/contenidos/pol_econ/documentos/Medicamentos_competencia.pdf 